Monika Dannerer (Innsbruck)

\title{
Die Universität als Vorbild/Spiegelbild/ Zerrbild für Spracheinstellungen und Sprachgebrauch heute?
}

\begin{abstract}
Der folgende Beitrag beschäftigt sich mit der Frage, wie weit der mündliche Sprachgebrauch an der Universität und die damit verbundenen Spracheinstellungen Vorbild, Spiegelbild oder aber Zerrbild des alltäglichen Sprachgebrauchs und allgemeiner Spracheinstellungen sind. Aus heuristischen Gründen liegt der Fokus dabei auf Sprachformen jenseits des Standards, die üblicherweise nicht mit einem universitären Sprachgebrauch in Verbindung gebracht werden. Die Datenerhebung dokumentiert exemplarisch die Situation an der Universität Salzburg, die Auswertung kombiniert in einem Mixed-Methods-Ansatz quantitative und qualitative Methoden.
\end{abstract}

\section{Einleitung}

Universitäten sind Institutionen der Forschung und der tertiären Bildung. Durch ihre regionale Verankerung und die gleichzeitig hohen Anreize für (großräumige) Mobilität entstehen für Lehrende, Studierende und Verwaltungspersonal Kontaktsituationen zwischen verschiedenen Erstsprachen und regionalen Varietäten. Diese Parameter prägen Spracheinstellungen und Sprachgebrauch und legen es nahe, sich in diesem Kontext mit der Standardsprache sowie der Fach- bzw. Wissenschaftssprache zu beschäftigen. Der vorliegende Beitrag hingegen gilt dem (berichteten) Gebrauch von und den Einstellungen zum Nonstandard, wobei als Nonstandard alle Sprachformen jenseits des Standards bzw. seiner arealen und nationalen Variation verstanden werden. ${ }^{1} \mathrm{Da}$ ein solcher Fokus auf universitären Sprachgebrauch ungewohnt ist und keine etablierte öffentliche Diskussion abbildet, können eher spontane Äußerungen individueller Spracheinstellungen erwartet werden.

1 Der Terminus „Substandard“, wie ihn z.B. Lenz (2003) in gleicher Bedeutung durchgehend verwendet oder wie ihn Löffler (2005, S. 11) zwischen Standard und Nonstandard ansetzt, wird vermieden, da er als abwertend missverstanden werden kann. 
Der Beitrag möchte im Hinblick auf die universitäre Sprachverwendung folgende Fragen beantworten:

1. Welche Rolle spielen - differenziert nach Akteur/innen und Situationen regionale Varietäten?

2. Welche Einstellungen haben die jeweiligen Gruppen dazu? Inwiefern spiegeln sich gesamtgesellschaftliche Befunde, wo wird eine Vorbildfunktion reklamiert, wo besteht die Gefahr, dass ein Zerrbild entsteht?

3. Welche Relevanz haben diese Befunde für das „heutige Deutsch“?

Nach einer terminologischen Klärung und Diskussion der Abgrenzungsproblematik der Varietäten v.a. im Hinblick auf die Wahrnehmung von Laien (Kap. 2) wird der Projektkontext erläutert, aus dem die Daten stammen (Kap. 3). Der Präsentation quantitativer und qualitativer Ergebnisse (Kap. 4 und 5) folgt abschließend die Diskussion, welches Bild sich daraus für das heutige Deutsch ergibt.

\section{Varietätenkonzeption und Forschungsmethodik}

Wenn Selbstaussagen von Proband/innen zu Sprachgebrauch und Spracheinstellungen analysiert werden, ist nicht nur die der Studie zugrunde liegende linguistische Terminologie darzulegen, sondern auch abzuklären, was die Proband/innen mit den Begriffen verbinden.

In der Linguistik wurde und wird Standardsprache als Sprache der Gebildeten definiert bzw. sogar spezifisch situational als Sprache in der Vorlesung (vgl. z.B. Steger 1968, S. 47). Auch für die gegenwärtige (gesprochene) Standardsprache wird - wenn man nicht überhaupt die Definition eines gesprochenen Standards ablehnt (vgl. die Diskussion in Hagemann/Klein/Staffeldt (Hg.) 2013) - als prototypisches Vorkommen der Kontext der Vorlesung genannt (Ammon et al. 2004, S. XXXVI, XL) bzw. allgemeiner die Schule (Barbour/Stevenson 1998, S. 145) oder „höhere kulturelle und Zivilisationsbedürfnisse“ (Daneš 2008, S. 2197). ${ }^{2}$

Untersuchungen zur (mündlichen) Sprachverwendung an der Universität stimmen mit dieser nicht nur schicht- sondern auch institutions-, situationsund diskurstypspezifischen Perspektive insofern überein, als sie viele Facetten

2 Selten finden sich demgegenüber Einschränkungen wie z.B. die von Auer (1997, S. 136), dass Orthoepie nur noch für Nachrichtensprecher/innen Prestige habe, nicht mehr jedoch „für [...] Universitätsprofessoren [...]“. Umgekehrt kommt Gebildeten weniger Relevanz als Vorbild für den Standard zu als geschulten Nachrichtensprecher/innen (vgl. Moosmüller 2015, S. 170). 
gesprochener Wissenschaftssprache - auch sprachkontrastiv - berücksichtigen, insgesamt jedoch kaum regionale Variation in den Blick nehmen (vgl. z.B. Biber 2006; ${ }^{3}$ Fandrych/Meißner/Slavcheva (Hg.) 2014).

Schichtspezifik bzw. Bildungsgrad haben auch in der Laienwahrnehmung Relevanz. Für Österreich verweist z.B. Moosmüller (2015, S. 177) darauf, dass als Standardsprecher/innen Personen identifiziert werden, die aus größeren kulturellen/politischen Zentren - v.a. Salzburg und Wien - stammen, keine südbairischen Merkmale und keine auffallenden dialektalen Eingabewechsel aufweisen und darüber hinaus über Bildung verfügen. Trotz dieser vermeintlichen Einhelligkeit ist die Grenze zwischen Standard und Nonstandardvarietäten im konkreten Fall und speziell auch über den gesamten deutschen Sprachraum hinweg keineswegs klar (vgl. z.B. Eisenberg 2017, S. 58).

Ganz ähnlich verhält es sich mit der Wahrnehmung der Sprecher/innen, was als Dialekt definiert werden kann. Sie schwankt zwischen ,jede Form des Sprechens, die irgendwelche standardabweichenden regionalen Merkmale enthält“ und Dialekten in einem linguistischen Verständnis (Schmidt 2017, S. 105). Dafür spielt nicht zuletzt die Sprachvarietät der unmittelbaren Umgebung eine wichtige Rolle. So stellen z.B. Plewnia/Rothe (2012, S. 16 f.) fest, dass in Regionen Deutschlands, in denen nur noch Regionalsprachen vorhanden sind (große Städte mit über 500.000 Einwohnern), jedes saliente Abweichen von einer empfundenen Standardvarietät als „Dialekt“ bezeichnet wird.

Nicht zuletzt werfen auch die „Sprachebenen dazwischen“, die in der Variationslinguistik zuletzt viel Aufmerksamkeit erhalten haben (vgl. z.B. Katerbow/ Werth (Hg.) 2010; Kehrein/Lameli/Rabanus (Hg.) 2015), ähnliche Probleme auf ${ }^{4}$ und zwar unabhängig davon, ob man eine Unterteilung des diaglossischen Kontinuums überhaupt ablehnt (Auer 2005, S. 22), ob man drei Stufen annimmt (z.B. Wiesinger 1997, S. 31; König/Elspaß/Möller 2015, S. 134), vier (z.B. Spiekermann 2005) oder aber sechs (z.B. Lenz 2003; Kehrein 2012, S. 33). Für die Wahrnehmung durch Sprecher/innen erhebt sich die Frage, wie weit diese überhaupt Stufen differenzieren (können).

Die (Fähigkeit zur) Unterscheidung von Varietäten, ihre Granularität und ihre Benennung sind auch in der Spracheinstellungsforschung ein relevantes for-

3 Biber erkennt große lexikalische wie syntaktische Unterschiede zwischen mündlicher und schriftlicher universitärer Sprachverwendung sowie zwischen den Fächern. Nonstandard thematisiert er hingegen nicht.

4 Eine Übersicht über die terminologische Vielfalt bietet u.a. Löffler (2005). Für Österreich sind u.a. detaillierte Ergebnisse aus dem SFB „Deutsch in Österreich“ (https://dioe.at; Stand: 12.5.2018) zu erwarten. 
schungsmethodisches Problem (vgl. z.B. Liebscher/Dailey-O’Cain 2009; Berthele 2010; Garrett 2010; Gärtig/Plewnia/Rothe 2010; Cuonz/Studler (Hg.) 2014; König 2014; de Cillia 2018).

Auch wenn Ergebnisse von Einstellungsstudien auf der einen Seite häufig relativ kongruent sind (vgl. Sieber 2013, S. 120), ${ }^{5}$ erschwert nicht nur der written language bias (Linell 2005) die Interpretation der Ergebnisse, sondern v.a. auch Unsicherheit und Unklarheit bezüglich Standardkonzeptionen und ihrer Benennungen v.a. in einem plurizentrischen/pluriarealen Kontext. ${ }^{6}$

Einen interessanten Lösungsansatz schlagen Christen et al. (2010) aufgrund von Erfahrungen mit Inkonsistenzen und vordergründig widersprüchlichen Äußerungen in Interviews vor: Sie gehen von unterschiedlichen, jeweils prototypischen mentalen Modellen der Standardkonzeption aus, über die Sprecher/innen verfügen bzw. auf die sie je nach Kontext flexibel referieren, und unterscheiden Hochdeutsch als
A) plurizentrische Sprachform (entsprechend dem eigenen Standardgebrauch),
B) normierte und kodifizierte Größe,
C) Sprachform der Zeitungen,
D) Sprachform von Radio und Fernsehen.
(vgl. ebd., S. 15 f.)

Diese mentalen Modelle werden von ihnen als nicht trennscharf gegeneinander abgrenzbar und überdies als offene Liste konzipiert.

\section{Datenmaterial und Methodik}

Die Datenbasis des Beitrags stammt aus dem Projekt VAMUS (= „Verknüpfte Analyse von Mehrsprachigkeiten am Beispiel der Universität Salzburg“), ${ }^{7}$ das eine gesamtuniversitäre Beschreibung und Analyse von Sprachgebrauch, Sprachein-

\footnotetext{
5 Dialekt wird - ähnlich wie die gesprochene Sprache insgesamt - attribuiert als „persönlich, vertraut, locker, frei, einfach, ausdrucksstark, sympathisch und lustig“, wohingegen Standardsprache und geschriebene Sprache als „unpersönlich, unvertraut, steif, kompliziert, wenig emotional, gepflegt und gehoben“ gelten.

6 Es ist anzunehmen, dass Befragte aus verschiedenen Regionen des deutschsprachigen Raumes und mit unterschiedlicher L1 divergierende Konzepte aufrufen.

7 Projektförderung durch den Jubiläumsfonds der Österreichischen Nationalbank (Projekt Nr. 15.827); Laufzeit: 4/2014-3/2018; Leitung Monika Dannerer und Peter Mauser. Zu Fragestellungen und Hypothesen vgl. u.a. Dannerer/Mauser (2016).
} 
stellungen und Sprachenpolitik unter Berücksichtigung des gesamten sprachlichen Repertoires (vgl. Busch 2012) zum Ziel hat. Dabei werden innere und äußere Mehrsprachigkeit (vgl. Wandruszka 1979) - d.h. Varietäten und Sprachen gemeinsam erfasst.

Die Universität Salzburg hat als Erhebungsort den Vorteil, dass von den rund 18.000 Studierenden zwar die größten Gruppen aus der unmittelbaren Umgebung, den Bundesländern Salzburg (41\%) und Oberösterreich (22\%), kommen, dass aber auch 20\% aus Deutschland stammen und 2\% deutschsprachige Südtiroler/innen sind. Neben der damit anzunehmenden Streuung der Varietäten divergieren auch die Erstsprachen: 7\% der Studierenden kommen aus dem nichtdeutschsprachigen Ausland (www.statistik.at).

Das Projekt verfolgt einen Mixed-Methods-Ansatz, der Online-Fragebögen, Interviews, schriftliche Dokumente sowie Aufnahmen aus Lehrveranstaltungen und Servicegesprächen verbindet und die Perspektiven verschiedener Gruppen an der Universität berücksichtigt. Die folgenden Analysen stützen sich auf zwei dieser Quellen und konzentrieren sich ausschließlich auf die innere Mehrsprachigkeit:

1. 1.227 Online-Fragebögen, die ca. 5\% aller Studierenden (S) ( $n=829), 10 \%$ der Lehrenden (L) (n=197) und 20\% der Verwaltungsmitarbeiter/innen (V) $(n=201)$ erfassen;

2. 123 ca. halbstündige Interviews mit den drei Gruppen zur thematischen Vertiefung.

Entsprechend der Größe der vier Fakultäten der Universität Salzburg stammen besonders viele Fragebögen aus der Kultur- und Gesellschaftswissenschaftlichen Fakultät (KGW), an zweiter Stelle aus den Naturwissenschaften (NW). Die Rechtswissenschaftliche (RW) und die Katholisch-Theologische Fakultät (KTH) spielen eine deutlich geringere Rolle (vgl. Tab. 1$){ }^{8}$

Als sprachlich prägenden „Herkunftsort“9 innerhalb des deutschsprachigen Raumes geben 25\% der teilnehmenden Studierenden, 24\% der Lehrenden und $6 \%$ der Verwaltungsmitarbeiter/innen ${ }^{10}$ einen Ort in Deutschland an. Für Auswertungen und Signifikanztests wurde für Deutschland aufgrund der im Süden höheren Präsenz von Dialekt im Alltag sowie der dort höheren Probandenzahl

8 In der Verwaltung erfolgt keine Auswertung nach Fakultäten, da hier übergreifende Kategorien deutlich prominenter vertreten sind.

9 Die Frage lautete: „Geburtsort oder der Ort, der Sie in Ihrer Kindheit sprachlich am stärksten geprägt hat".

10 Diese Gruppe ist daher nicht sinnvoll statistisch differenzierbar. 
und damit möglichen Differenzierungen zwischen Bayern (BAY), Baden-Württemberg (BW) und den anderen Bundesländern (stark vergröbernd zusammengefasst als „Mittel- und Norddeutschland“ (MND)) unterschieden.

Im Hinblick auf die Erstsprachen (L1) und den Zweitspracherwerb wurden drei Gruppen gebildet: Proband/innen mit

- Deutsch als (einer ihrer) L1/Muttersprache(n) (DaM);

- einer anderen L1 als Deutsch und Schulabschluss im deutschsprachigen Raum (DaZ);

- $\quad$ einer anderen L1 und Schulabschluss außerhalb des deutschsprachigen Raumes (DaF).

Tab. 1: Differenzierungen nach Fakultät, Herkunft und $\mathrm{L}^{11}$

\begin{tabular}{lccc}
\hline Gruppierung/Anzahl & Studierende & Lehrende & Verwaltung \\
\hline Gesamt & 829 & 197 & 201 \\
\hline KGW & 368 & 88 & 32 \\
\hline NW & 198 & 48 & 20 \\
\hline RW & 57 & 26 & 16 \\
\hline KTH & 12 & 9 & 8 \\
\hline Österreich (AUT) & 381 & 103 & 125 \\
\hline Bayern (BAY) & 89 & 10 & 2 \\
\hline Baden-Württemberg (BW) & 14 & 6 & 1 \\
\hline Mittel-/Norddeutschland (MND) & 31 & 17 & 6 \\
\hline DaM & 591 & 156 & 149 \\
\hline DaZ & 33 & 3 & 6 \\
\hline DaF & 62 & 16 & 11 \\
\hline
\end{tabular}

Interviews wurden mit Personen geführt, die sich im Fragebogen dazu bereit erklärt hatten, wobei bei den Studierenden ausgewählt und auf eine möglichst gute Streuung geachtet werden konnte.

11 Differenzen zur Gesamtsumme ergeben sich aus unvollständig ausgefüllten Fragebögen. Die jeweiligen Angaben waren keine Pflichtfelder. 


\section{Ergebnisse quantitativ}

\subsection{Angaben zur Varietätenverwendung}

Der Fragebogen enthielt die Frage „In welcher Situation verwenden Sie selbst die folgenden Varietäten (= Sprechweisen) des Deutschen (Standardsprache/ Umgangssprache/Dialekt)? Kreuzen Sie an - mehrere Antworten sind möglich. Hinweis: Die Umgangssprache liegt zwischen Standardsprache und Dialekt.“

Die vorgegebenen Situationen variierten bei den einzelnen Gruppen (S/L/V), umfassten jedoch immer sowohl Situationen in der Herkunftsfamilie/im Freundeskreis als auch formelle Situationen an der Universität. Für die Varietäten wurden drei Formen differenziert (Standardsprache, Umgangssprache, Dialekt), um die Proband/innen nicht zu überfordern. Durch die Möglichkeit von Mehrfachantworten konnte die tatsächliche Variationsbreite statt einer fiktiven Mischform angegeben werden, was auch häufig genutzt wurde (vgl. Vergeiner i.Vorb.).
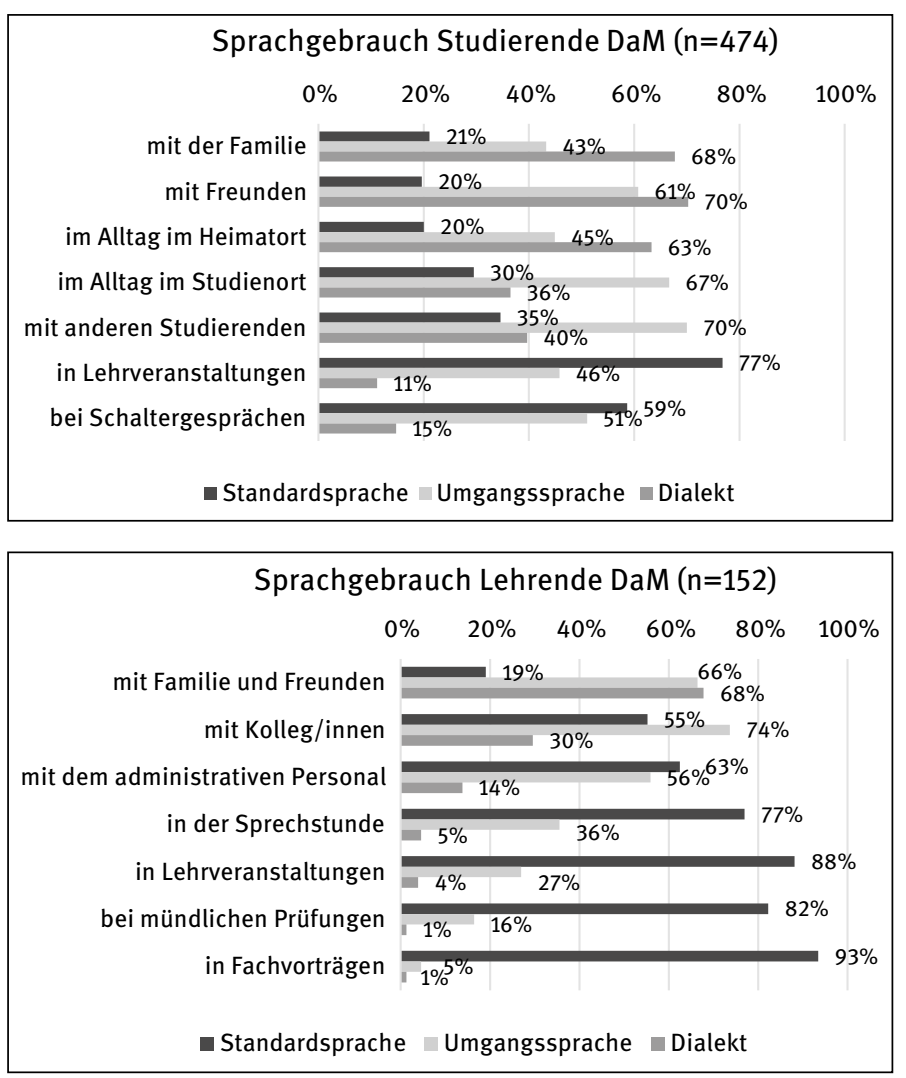


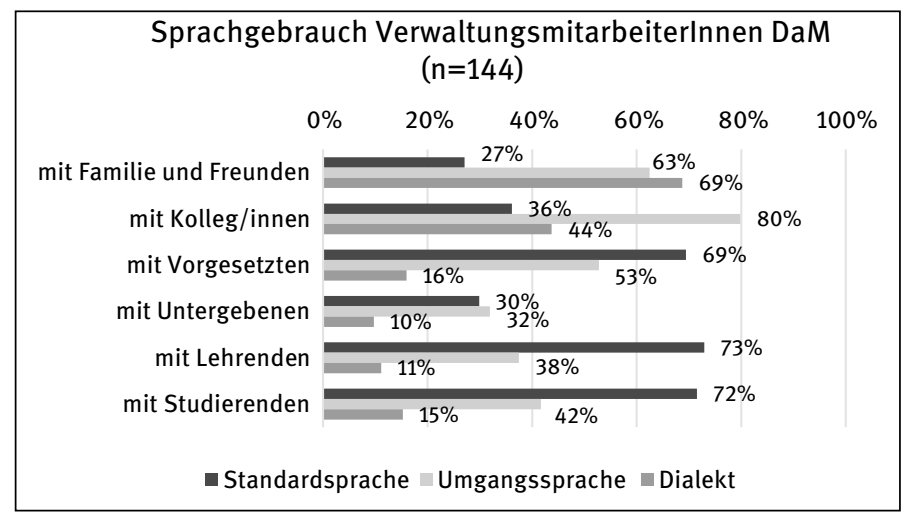

Abb. 1-3: Sprachgebrauch von Studierenden, Lehrenden, Verwaltungsmitarbeiter/innen

Das Gesamtbild zeigt, dass nach Angaben der Proband/innen „Dialekt“ im Privaten (am Heimatort) eindeutig dominiert. An der Universität hingegen geben alle drei Gruppen an, dominant Standard zu verwenden, wenn auch die Ausprägungen z.T. unterschiedlich sind. Beispielsweise verwenden 59\% der Studierenden und 63\% der Lehrenden, die diese Frage beantwortet haben, mit dem administrativen Personal Standardsprache, demgegenüber sprechen 72\% bzw. 73\% der Verwaltungsmitarbeiter/innen im Kontakt mit Studierenden bzw. Lehrenden Standard. ${ }^{12}$ Insgesamt spielt Nonstandard in verschiedenen Kontexten eine z.T. gewichtige Rolle. Bei aller gebotenen Vorsicht im Hinblick auf die Varietätenunterscheidung durch die Proband/innen (vgl. Kap. 5.1) wird damit eine sehr klare institutions-, aber v.a. situationsspezifische Differenzierung erkennbar.

Innerhalb der Probandengruppen gibt es v.a. nach Fakultät und regionaler Herkunft signifikante Unterschiede, wobei die entsprechenden Tests aufgrund der Zellbesetzung nur für Lehrende und Studierende möglich sind. ${ }^{13}$ In beiden Fällen weisen die Studierenden deutlich mehr signifikante Unterschiede als die Lehrenden auf. Signifikante Fakultätsunterschiede zeigen sich v.a. zwischen Studierenden bzw. Lehrenden der RW, die in vielen Situationen (einschließlich der

12 Dieser Befund spiegelt sich in den qualitativen Aussagen zur Rolle von Hierarchie und institutioneller Funktion (vgl. Kap. 5.2).

13 Alle Tests wurden mittels Kreuztabelle berechnet; bei $\mathrm{df}=1$ wurde mit der Korrektur nach Yates gearbeitet; ansonsten wurde Chi-Quadrat nach Pearson verwendet. Aufgrund der hohen Belegzahlen (meist weit über 20) und der insgesamt meist guten Zellbesetzungen können fast alle Ergebnisse als hinreichend robust gelten. 
privaten) eher zum Standard tendieren, und denjenigen der NW und der KTH, die eher nonstandardaffin sind. Bei den Studierenden beziehen sich signifikante Unterschiede ausschließlich auf den privaten Bereich, auf Gespräche mit anderen Studierenden und Verwaltungsgespräche. Bei den Lehrenden sind vom Gespräch mit Kolleg/innen bis zum Fachvortrag unterschiedliche berufsbezogene Situationen betroffen.

Besonders viele signifikante Unterschiede sind nach „Herkunftsregion“ zwischen Proband/innen aus Österreich ${ }^{14}$ und den verschiedenen Regionen Deutschlands festzustellen ${ }^{15}$ (vgl. Tab. 2): Studierende (S) aus Österreich (AUT) verwenden in der Familie, mit Freunden und am Heimatort eher Dialekt (D), am Studienort, in der Lehrveranstaltung (LV) und bei Gesprächen mit der Verwaltung (Verw) eher Umgangssprache (Ugs). Studierende aus BAY hingegen sind in informellen Situationen signifikant offener für die Standardsprache (St) als die Studierenden aus AUT. Ähnlich, wenn auch nicht ganz so ausgeprägt, verhält es sich zwischen Studierenden aus AUT und BW, sehr systematisch zwischen solchen aus AUT und MND: AUT-Studierende verwenden nicht nur in informellen Kontexten, sondern auch in LVs und mit der Verwaltung mehr Dialekt, in LVs und am Schalter auch mehr Umgangssprache. Demgegenüber verwenden die MNDStudierenden in allen Situationen mehr Standard und informell auch mehr Umgangssprache. Nicht zu übersehen sind auch die signifikanten innerdeutschen Unterschiede zwischen BAY und MND, die auch in LVs und mit der Verwaltung gelten: Die MND-Studierenden wählen immer signifikant mehr Standard, die aus BAY signifikant mehr Dialekt.

An der Universität spiegelt sich also die gesamtgesellschaftlich stärkere Dialektverwendung im süddeutschen Sprachraum. Ob die Tatsache, dass sie bei Studierenden nicht nur im Privaten, sondern auch an der Universität stärker ausgeprägt ist als bei Lehrenden, mit „institutionellem Novizentum“ in Verbindung gebracht werden kann oder ob sich eine Ausweitung der Domänenspezifik des Nonstandards abzeichnet, lässt sich aus dem Material heraus nicht beantworten. Die erheblichen situativen Unterschiede zeigen überdies, dass Varietätenkontakt am Studienort und die Institution an sich einen großen Einfluss haben, auch wenn ,universitärer Sprachgebrauch“ offenbar nicht in allen Bereichen gleichgesetzt werden kann mit der Verwendung der Standardsprache.

14 Die Bezeichnungen werden hier verkürzt verwendet und sind zu lesen als „Personen, die sprachlich am stärksten in X geprägt wurden“.

15 Das bedeutet nicht, dass es innerhalb Österreichs keine signifikanten Unterschiede geben würde - etwa zwischen den stärker dialektaffinen Oberösterreicher/innen und den Salzburger/ innen (vgl. Vergeiner i.Vorb.). 
Tab. 2: Signifikante regionale Differenzen im Sprachgebrauch: Studierende (recte) und Lehrende (kursiv) ${ }^{16}$

\begin{tabular}{|c|c|c|c|c|}
\hline & AUT & BAY & BW & MND \\
\hline $\begin{array}{l}\text { AUT im } \\
\text { Vergleich } \\
\text { zu }\end{array}$ & $x$ & 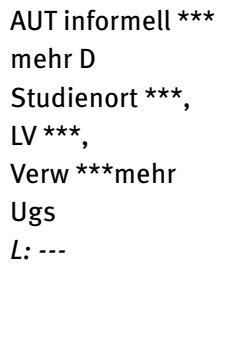 & $\begin{array}{l}\text { AUT informell } * \star \\
\text { mit and. } S^{\star \star \star} \\
\text { mehr D; } \\
\text { Studienort *, } \\
\text { LV } * \star \star \text {, Verw } * \star \star \\
\text { mehr Ugs } \\
\text { L: --- }\end{array}$ & 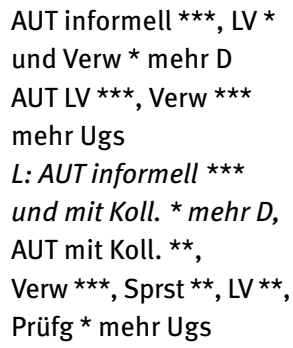 \\
\hline $\begin{array}{l}\text { BAY im } \\
\text { Vergleich } \\
\text { zu }\end{array}$ & $\begin{array}{l}\text { BAY informell *** } \\
\text { mehr St und Ugs } \\
\text { L: BAY Prüfg *, } \\
\text { Vortr * mehr D }\end{array}$ & $x$ & $\begin{array}{l}\text { BAY im } \\
\text { Heimatort * } \\
\text { mehr St, } \\
\text { am Studien- } \\
\text { ort **, mit S ** } \\
\text { mehr D } \\
\text { L: --- }\end{array}$ & 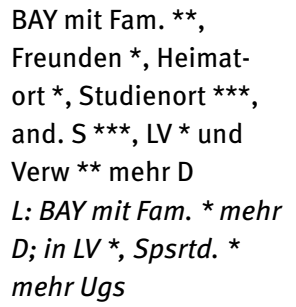 \\
\hline $\begin{array}{l}\text { BW im } \\
\text { Vergleich } \\
\text { zu }\end{array}$ & $\begin{array}{l}\text { BW informell *, } \\
\text { Verw ** } \\
\text { Studort } * \star \star \\
\text { mehr St; } \\
\text { mit Freunden ** } \\
\text { mehr Ugs } \\
\text { L: --- }\end{array}$ & $\begin{array}{l}\text { S.o. } \\
\text { L: --. }\end{array}$ & $x$ & $\begin{array}{l}\text { BW im Heimatort * } \\
\text { mehr D } \\
\text { L: --- }\end{array}$ \\
\hline $\begin{array}{l}\text { MND im } \\
\text { Vergleich } \\
\text { zu }\end{array}$ & 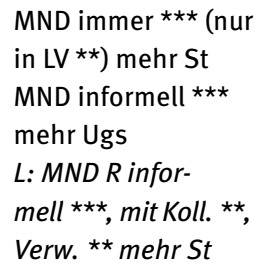 & $\begin{array}{l}\text { MND mit Fam. }{ }^{\star \star \star} \text {, } \\
\text { Freunden }{ }^{\star \star} \text { and. } \\
\text { Stud }{ }^{\star \star}, \text { sonst } \\
\text { immer }{ }^{*} \text { mehr St } \\
\text { L: MND mit } \\
\text { Fam. }{ }^{\star \star} \text {, mit } \\
\text { Koll. }{ }^{\star \star} \text { mehr St }\end{array}$ & $\begin{array}{l}\text { MND mit Fam. **, } \\
\text { Freunden *, am } \\
\text { Heimatort ** } \\
\text { mehr St } \\
\text { L: MND mit } \\
\text { Fam. *, mit Koll. * } \\
\text { mehr St }\end{array}$ & $x$ \\
\hline
\end{tabular}

16 Für die Signifikanzniveaus gilt: ${ }^{\star \star}=$ höchst signifikant $(\mathrm{p}<0-0,001),{ }^{\star \star}=$ hoch signifikant $(0,001<\mathrm{p}>0,01),{ }^{\star}=$ signifikant $(0,01<\mathrm{p}>0,05)$. 


\subsection{Angemessenheitsurteile}

Im Fragebogen wurden auch Angemessenheitsurteile erhoben. Die Frage lautete bei den Studierenden: „Welche Sprechweisen des Deutschen finden Sie in welcher Situation angemessen? Kreuzen Sie an - mehrere Antworten sind möglich.“ Bei den Lehrenden wurden komplementär Angemessenheitsvorstellungen im Hinblick auf studentisches sprachliches Verhalten elizitiert ${ }^{17}$ („Welche Varietäten des Deutschen halten Sie in Beiträgen von Studierenden für angemessen?“).

Regionale Unterschiede weisen dabei in die gleiche Richtung, sind jedoch weniger häufig signifikant (die binnendeutschen Unterschiede fallen z.B. fast gänzlich weg), was bedeutet, dass eine gewisse Toleranz über den eigenen Sprachgebrauch hinaus die Konturen unschärfer werden lässt.

In dieser Frage sind Studierende mit DaF klar von Studierenden mit DaM und $D a Z^{18}$ unterscheidbar: Umgangssprache und Dialekt sind für sie auch in informellen Situationen signifikant weniger angemessen, im Hinblick auf die Umgangssprache gilt dies auch für LVs und Schaltergespräche. Beide Gruppen sind sich jedoch einig über die hohe Angemessenheit der Standardsprache und die geringe Angemessenheit des Dialekts in diesen institutionellen Settings.

\section{Ergebnisse qualitativ}

Ergänzend zu den Fragebögen zeigen sich in den Interviews Konzeptionen von und Einstellungen zu Varietäten im Allgemeinen sowie Bewertungen und Begründungen von Standard- und Nonstandardverwendung im spezifisch universitären Kontext. Auch hier ist zu fragen, wie weit sich gesamtgesellschaftliche Auffassungen spiegeln oder ob institutionsspezifische Argumentationen auftreten.

\subsection{Standardkonzeptionen}

Die Daten erlauben nicht nur einen Einblick in verschiedene Konzeptionen von Standard und Nonstandard, sie sind ohne diese Einblicke auch nicht interpretierbar.

17 Differenziert wurden hier z.B. Wortmeldungen, Präsentationen und Prüfungen.

18 Studierende mit DaZ unterscheiden sich in keinem Punkt signifikant von denjenigen mit DaM. 
Monozentrische Auffassungen einer Standardsprache werden besonders häufig von Sprecher/innen mit DaF sowie von Proband/innen aus MND vertreten. Sie kommen verhältnismäßig häufig zum Schluss, dass niemand in Österreich Standard spreche, man höre immer eine Differenz zur Standardsprache - auch die Professor/innen haben zum Teil einen „Akzent“ (SEF $\left.{ }^{19}\right)$. Diese monozentrischen Positionen führen so weit, dass selbst die eigene Varietät hinterfragt wird, wenn die Sprache in Österreich erworben wurde (LUE). ${ }^{20}$ Auch Sprecher/innen aus Österreich vertreten z.T. eine monozentrische Perspektive, wenn sie etwa den Standard in Hannover, Berlin, Hamburg oder allgemeiner „im Norden“ Deutschlands verorten. ${ }^{21}$

Als Ursache für die Verwendung von Nonstandardvarietäten an der Universität wird (u.a. aus einer monozentrisch geprägten Annahme heraus) ein prinzipielles Unvermögen, das eigene, landschaftlich geprägte Idiom zu verlassen, angenommen. V.a. Befragte mit nicht-deutscher L1 bringen sie auch mit Faulheit, Unwillen oder gar Arroganz in Verbindung bzw. im spezifisch akademischen Kontext kommt es sogar zu einer Gleichsetzung mit fehlender fachlicher Präzision. Auch Schichtspezifik und mangelnde Konsequenz der österreichischen Schule beim Einfordern der Standardsprache werden angeführt.

Wenn verschiedene Standardvarietäten angenommen werden, so finden sich plurizentrische und - etwas weniger häufig - pluriareale Konzeptionen, die sowohl Regionen in Deutschland als auch Regionen in Österreich differenzieren. In mehreren Interviews wird die gegenseitige Wahrnehmung im universitären Kontext angesprochen: Sowohl eine Differenz zwischen der Eigen- und Fremdwahrnehmung in unterschiedlichen Umgebungen (LIG) als auch die berichteten bzw. vermuteten Einstellungen der anderen über die eigene Varietät sind dabei Thema. Die Verwaltungsmitarbeiterin VAB meint etwa, dass das „österreichische Idiom“

19 Der erste Buchstabe der Sigle bezeichnet die Gruppe (L/S/V), die beiden anderen sind pseudonymisierte Initialen.

20 Vgl. Ransmayr (2006) zu Vorbehalten gegenüber österreichischen Lektor/innen im Sprachunterricht an Auslandsgermanistiken.

21 Die Granularität der Verortung bzw. die Bezeichnung von Varietäten ist sehr unterschiedlich (vgl. Lameli/Purschke/Kehrein 2008; Purschke 2010) und schwankt zwischen Großregionen (Bairisch/Bayrisch), Regionsbezügen (Niederbayrisch) und Ortsbezügen (Berlinerisch, Hamburgisch, Hannoveranisch). Erstaunlicherweise geht sie auch in Österreich nur selten unter die Verwaltungseinheit „Bundesland“. In Salzburg nennt z.B. LIC „Innergebirg“ (Salzburg südlich des Pass Lueg, also Pongau, Pinzgau und Lungau umfassend - eine alte, aber immer noch gängige Bezeichnung), daneben treten „Pinzgauerisch“ (LEI, LAF, VED, VOA, SUD, SUH), „Pongauerisch“ (LIH, SUH) und „Lungauerisch“ (SEA) auf, in Oberösterreich „Innviertlerisch“ (LAE, VAD, VOD) oder Mühlviertlerisch (LIH), für den 16. Wiener Gemeindebezirk „Ottakringerisch“ (SIB). 
in professionellen Kontexten in Deutschland als „entzückend“ oder „süß“ wahrgenommen werde, dass man sich dabei aber nicht immer ernst genommen fühle (09:12-10:16).

Neben Proband/innen mit einem sehr konsistenten Konzept von Standardsprache (z.B. Proband/innen mit DaF) gibt es viele, die - wie von Christen et al. (2010) beschrieben - je nach Gesprächskontext auf unterschiedliche Konzepte rekurrieren. Dies deutet darauf hin, dass der Standardbegriff ausgehandelt werden muss, weil er je nach Kontext unterschiedliche Bezugspunkte haben kann und jeweils andere soziale Gruppen und Regionen relevant werden.

\subsection{Bewertungen und Begründungen von Standard- und Nonstandardverwendung}

In den Interviews wird der Nonstandard(-gebrauch) häufiger als der Standard(-gebrauch) thematisiert. Die Antworten lassen sich in vier Kategorien zusammenfassen: 1) Individuum, 2) Ästhetik, 3) Funktion und 4) Allgemeines.

Im Hinblick auf das Individuum (1) wird Nonstandard wie auch in anderen Kontexten mit Authentizität, Identität, Natürlichkeit und Diversität verbunden in seltenen Fällen wird die Umkehrung dieser Nennungen auch als Gegenargument gegen die Standardsprache eingesetzt. Es fällt auf, dass diese Argumente ausschließlich von österreichischen und bayrischen Proband/innen stammen.

Ästhetik (2) wird für den Dialekt fast nur von österreichischen Proband/innen reklamiert (Klangerlebnis, „Farbe“), für die Standardsprache auch von Personen mit DaF („schön“). Während Kritik am Dialekt nie mit ästhetischen Urteilen verbunden ist, wird Standardsprache nicht nur als „schönstes Hochdeutsch“ apostrophiert, sondern auch als „brav“, „steril“ oder „hochgestochen“ abgelehnt. ${ }^{22}$

Positive funktionale Argumente (3) beziehen sich für den Dialekt sehr unspezifisch v.a. auf die Möglichkeit der Differenzierung sozialer Beziehungen. Nur sehr vereinzelt werden Zweifel an seiner Funktionalität für den Ausdruck von wissenschaftlichen Inhalten geäußert (vgl. aber SOJ, SUH). Im Rahmen der dabei verwendeten Argumentation wird allerdings das Dilemma der unklaren Reichweite verwendeter Termini deutlich. LEA beispielsweise, eine aus Süddeutschland stammende Lehrende an der KGW, beklagt zunächst exemplarisch die ,dialektale Art der Relativsatzbildung“ (der was), die in formellen Kontexten vermieden werden solle (04:10-4:52). Sie klagt, dass es Kolleg/innen gebe, die „starken Dialekt

$22 \mathrm{Zu}$ z.T. sehr ähnlichen semantischen Topoi der Varietätenbenennung vgl. z.B. Christen (2010) und Tophinke/Ziegler (2014). 
sprechen“ - nicht „österreichischen Dialekt“, sondern „,andere Dialektarten“, wie sie sofort präzisiert (Beispiel (1), Z. 002). Ihre Forderung, man solle sich auf internationalen Konferenzen um „Standardnähe“ bemühen, lässt das Konzept eines diaglossischen Kontinuums erkennen (Z. 004). Auf das signalisierte Verstehen der Interviewerin, dass ja ansonsten die Verständlichkeit leiden würde, widerspricht sie jedoch, dass es doch nicht um Dialekt gehe: ${ }^{23}$

Beispiel (1) LEA_KGW_DaM - Aufnahme: 15.01.2015, Länge: 32:00 Ausschnitt: 05:21-05:49

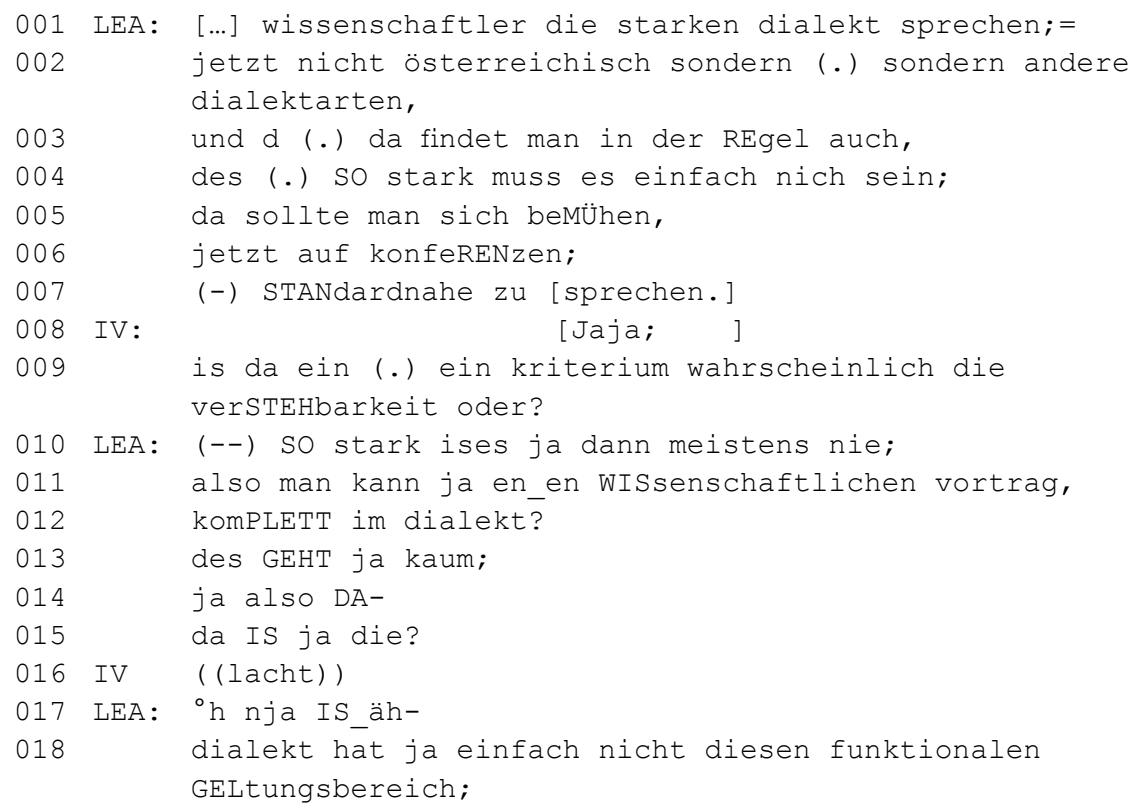

Offen bleibt hier, was zunächst unter „starkem Dialekt“ gemeint war - die Negation „nicht österreichisch“ deutet eher auf einen Regiolekt oder sogar auf eine nationale Standardvarietät hin. Mit der Korrektur, dass ein Vortrag nicht „komplett im Dialekt“ sein könne (Z. 011-013), da Dialekt diese Funktionen nicht erfülle, kann sie letztlich keine Klarheit schaffen.

Auch Argumente für die Standardsprache werden nur sehr selten mit der Zuschreibung einer anderen Ausdrucksmöglichkeit verbunden - etwa wenn sie

23 Der Aspekt der kommunikativen Reichweite wird für den Standard nur im Hinblick auf die Studierenden mit anderer L1 als Deutsch angeführt - interessanterweise ausschließlich von deutschen Proband/innen und solchen aus nichtdeutschsprachigen Ländern. 
als „detaillierter“, „genauer“, „gezielter“, „deutlicher“ (SEA) und deshalb v.a. in der Wissenschaft als angemessener dargestellt wird. In diesen Fällen werden häufig Standardsprache und Fachsprache vermengt - diese Zuordnungen finden sich übrigens sowohl von Österreicher/innen als auch von Deutschen und DaFSprecher/innen. Häufig wird dabei darauf verwiesen, dass es professioneller „wirkt“ (SUA), d.h. nicht so sehr auf die tatsächliche Professionalität bzw. Funktionalität der Standardvarietät. Überdies wird die Nähe zur Schriftlichkeit als didaktischer Vorteil (z.B. für Studierende) gesehen (LED, VUB, SAI).

Nicht funktionale, sondern allgemeine Urteile (4) sehen Nonstandard als unangemessen (LOF, VEB) v.a. bei Präsentationen und Prüfungen, aber auch bei Sprechstundengesprächen (SOJ) oder Wortmeldungen vor Gruppen (VAB). V.a. deutsche Lehrende vermuten für Nonstandardsprecher/innen explizit „Wettbewerbsnachteile“, d.h. eine ggf. schlechtere Beurteilung. SEK, ein KGW-Studierender aus Italien, fasst dies als universelle Normanforderung zusammen, in der er universitären Sprachgebrauch mit Schriftlichkeit und dem Streben nach der „höchsten“ Varietät einer Sprache verbindet, deren Verwendung wiederum ihrerseits das Niveau einer Universität anzeige bzw. hebe.

In wenigen Fällen wird die Forderung nach dem Standard auch mit künftiger Berufstätigkeit (z.B. als Lehrer/in; SOF) und/oder künftiger Mobilität (SID) begründet.

Jenseits des Sprachgebrauchs in den Lehrveranstaltungen finden sich mehrfach Belege in den Interviews von Studierenden und Verwaltungspersonal, dass Standardverwendung nicht nur eine wichtige Form der Rücksichtnahme gegenüber Personen mit anderen Erstsprachen als Deutsch ist (z.B. VID, VUD, VOB, SIB, SIC), ${ }^{24}$ sondern ganz allgemein als angemessen in der Kommunikation mit Höhergestellten (Älteren oder Hierarchiehöheren) gewertet wird. Es wird auf eine soziale Norm referiert, die als konsensuell dargestellt wird: Es „gehört sich eben“, Standard zu sprechen (SUI, SEA, SAI), anderes wird mit „Unhöflichkeit“ oder mangelndem Respekt in Verbindung gebracht (SEK).

Mit der „institutionellen Rolle“ argumentieren auch Verwaltungsmitarbeiter/ innen. Mehrfach wird angeführt, man solle ,als offizielle Person“ gegenüber Studierenden und Lehrenden (SEH) Standardsprache verwenden (VIC). Begründet wird dies z.T. mit mehr Klarheit (VIC, VIA).

24 Wenn von Höherrangigen eingefordert wird, sie mögen Standard verwenden, wird ausschließlich die Verständlichkeit für Studierende mit anderer L1 als Deutsch als Argument genannt. Damit wird selbst im Interview Kritik, dass nicht institutions- oder rollenangemessenen gesprochen würde, vermieden. 
Bei den Proband/innen aus der Gruppe DaF fällt generell auf, dass sie den Standardgebrauch überwiegend in Verbindung mit der Bewertung von Varietäten und Varietätengebrauch in vergleichbaren Situationen im Herkunftsland bzw. der L1 beurteilen. Daneben scheinen Dauer des Kontakts und Varietätenkompetenz im Deutschen eine Rolle zu spielen: Positive Urteile fällen eher Personen, die bereits länger in Österreich sind und/oder Varietäten gut verstehen. ${ }^{25}$ Diese Parameter gelten offenbar ähnlich für Lehrende aus Deutschland: Sie scheinen mit zunehmender Aufenthaltsdauer in Österreich eher einen plurizentrischen oder pluriarealen Standard wahrzunehmen. So spricht LIF, der aus MND kommend seit ca. 10 Jahren in Österreich ist, seine Hemmungen an, österreichische Studierende sprachlich zu kritisieren. Zum einen möchte er nicht „von oben herab“ wirken, zum anderen betont er, dass es „ein österreichisches Hochdeutsch gibt“, das auch für professionelle Kontexte „hervorragend“ geeignet sei, sodass „kein anderes Hochdeutsch“ nötig sei, um „Kompetenz in Österreich zu zeigen“. Dies impliziert zum einen die Existenz nationaler Standardvarietäten und zum anderen, dass man sich außerhalb Österreichs - möglicherweise auch als Österreicher/in besser einer anderen Standardvarietät bedienen solle.

\section{Fazit}

Die im Beitrag herausgearbeiteten Ergebnisse lassen im Hinblick auf Sprachgebrauch und Spracheinstellungen an der Universität folgende Perspektivierungen auf das heutige Deutsch zu:

Universitärer Sprachgebrauch als Vorbild:

1. Im Durchschnitt dominiert im universitären Kontext die Standardsprache deutlich, was v.a. im süddeutschen Sprachraum eine klare Differenz zur Alltagssprachverwendung bedeutet.

2. Standardsprache wird häufig als sozial angemessen/notwendig im institutionellen hierarchischen Gefüge (Höflichkeit) und in Relation zur Gesamtgesellschaft (,wer, wenn nicht die Universität“) angesehen.

3. Eine explizite Argumentation im Hinblick auf wissenschaftliche Anforderungen an sprachliche Präzision tritt nur selten auf.

25 Dazu passt, dass die Gruppe DaZ sehr ähnlich wie monolingual deutschsprachige Proband/ innen (DaM) urteilt. 
Universitärer Sprachgebrauch als Spiegelbild:

4. Für den Alltag geben auch die Universitätsangehörigen eine intensive Verwendung von Dialekt und Umgangssprache an und lehnen Standardsprache sehr häufig als „unangemessen“ ab.

5. Je formeller eine Situation ist, desto eher wird aber Standardsprache gesprochen und als angemessen empfunden.

Universitärer Sprachgebrauch als Zerrbild:

6. Durch die z.T. sehr großen Fakultätenunterschiede könnte die KGW nur ein verzerrtes Bild für die gesamte Universität liefern.

7. Durch den institutionsspezifisch hohen written language bias wie durch den mobilitätsbedingt intensiven Varietätenkontakt kommt es möglicherweise zu einer Verzerrung der Beurteilung des eigenen Sprachgebrauchs im Sinne eines stärker monozentrischen Bildes einer Standardsprache.

Die Universität ist ein spannendes Labor für Sprachen- und Varietätenkontakt. Dazu tragen nicht nur regionale Verankerung, Internationalität und inhaltlich komplexe Anforderungen bei, sondern auch Parameter, auf die in diesem Beitrag nicht eingegangen werden konnte, wie z.B. die Zunahme informeller Interaktion (z.B. mehr spontane E-Mail-Kommunikation, weniger schriftlich ausformulierte Vorlesungen), sowie ein - zumindest auf der Leitungsebene - relativ klar umrissenes Selbstverständnis als führende Bildungsinstitution, an der nur Standardsprache Platz haben sollte. Es wurde deutlich, dass Nonstandardvarietäten zumindest in bestimmten universitären Situationen Verwendung und auch Akzeptanz finden. Ob hier von einer Ausweitung der Verwendungsdomänen von Nonstandard oder gar von einer Destandardisierung $\mathrm{zu}$ sprechen ist, scheint jedoch nicht zuletzt in Anbetracht fehlender Vergleichsuntersuchungen noch nicht entscheidbar.

\section{Literatur}

Ammon, Ulrich et al. (2004): Variantenwörterbuch des Deutschen. Die Standardsprache in Österreich, der Schweiz und Deutschland sowie in Liechtenstein, Luxemburg, Ostbelgien und Südtirol. Berlin/New York.

Anders, Christina Ada/Hundt, Markus/Lasch, Alexander (Hg.) (2010): Perceptual dialectology. Neue Wege der Dialektologie. 2. Aufl. (= Linguistik - Impulse und Tendenzen 38). Berlin.

Auer, Peter (1997): Führt Dialektabbau zur Stärkung oder Schwächung der Standardvarietät? Zwei phonologische Fallstudien. In: Mattheier, Klaus J./Radtke, Edgar (Hg.): Standardisierung und Destandardisierung europäischer Nationalsprachen. (= VarioLingua 1). Frankfurt a.M., S. 129-161. 
Auer, Peter (2005): Europe's sociolinguistic unity, or: A typology of European dialect/standard constellations. In: Delbecque, Nicole/van der Auwera, Johan/Geeraerts, Dirk (Hg.): Perspectives on variation. Sociolinguistic, historical, comparative. (= Trends in Linguistics. Studies and Monographs 163). Berlin, S. 7-42.

Barbour, Stephen/Stevenson, Patrick (1998): Variation im Deutschen. Soziolinguistische Perspektiven. Berlin.

Berthele, Raphael (2010): Der Laienblick auf sprachliche Varietäten: Metalinguistische Vorstellungswelten der Deutschschweizerinnen und Deutschschweizer. In: Anders/Hundt/ Lasch (Hg.), S. 245-267.

Biber, Douglas (2006): University language. A corpus-based study of spoken and written registers. (= Studies in Corpus Linguistics 23). Amsterdam.

Busch, Brigitta (2012): The linguistic repertoire revisited. In: Applied Linguistics 33, 5, S. 503-523.

Christen, Helen (2010): Was Dialektbezeichnungen und Dialektattribuierungen über alltagsweltliche Konzeptualisierungen sprachlicher Heterogenität verraten. In: Anders/Hundt/ Lasch (Hg.), S. 269-291.

Christen, Helen et al. (2010): Hochdeutsch in aller Munde. Eine empirische Untersuchung zur gesprochenen Standardsprache in der Deutschschweiz. (= ZGL-Beiheft 140). Stuttgart.

de Cillia, Rudolf (2018): Sprache(n) im Klassenzimmer. Varietätengebrauch und Spracheinstellungen in der Schule aus Sicht der Lehrer/innen und der Schüler/innen. In: Dannerer, Monika/Mauser, Peter (Hg.): Formen der Mehrsprachigkeit. Sprachen und Varietäten in sekundären und tertiären Bildungskontexten. (= Stauffenburg Linguistik 102). Tübingen, S. 67-85.

Cuonz, Christina/Studler, Rebekka (Hg.) (2014): Sprechen über Sprache. Perspektiven und neue Methoden der Spracheinstellungsforschung. (= Stauffenburg Linguistik 81). Tübingen.

Daneš, František (2008): Herausbildung und Reform von Standardsprachen und Destandardisierung. In: Ammon, Ulrich et al. (Hg.): Sociolinguistics. Soziolinguistik. 2., vollst. neu bearb. u. erw. Aufl. Bd. 3. Berlin, S. 2197-2209.

Dannerer, Monika/Mauser, Peter (2016): Österreichische Universitäten als mehrsprachige Interaktionsräume? Universitäre Sprachenpolitik vor dem Hintergrund des Projektes „Verknüpfte Analyse von Mehrsprachigkeiten am Beispiel der Universität Salzburg (VAMUS)“. In: Jabłkowska, Joanna/Kupczyńska, Kalina/Müller, Stephan (Hg.): Literatur, Sprache und Institution. (= Stimulus 2014). Wien, S. 170-183.

Deutsche Akademie für Sprache und Dichtung/Union der Deutschen Akademien der Wissenschaften (Hg.) (2006/2017): Vielfalt und Einheit der deutschen Sprache. Zweiter Bericht zur Lage der deutschen Sprache. Unter Mitarbeit von Heinrich Detering und Hanns Hatt. Tübingen.

Eichinger, Ludwig M./Kallmeyer, Werner (Hg.) (2005): Standardvariation. Wie viel Variation verträgt die deutsche Sprache? (= Jahrbuch des Instituts für Deutsche Sprache 2004). Berlin/New York.

Eisenberg, Peter (2017): Standarddeutsch. Überdachung der Varietäten. In: Deutsche Akademie für Sprache und Dichtung/Union der Deutschen Akademien der Wissenschaften (Hg.), S. 53-104.

Fandrych, Christian/Meißner, Cordula/Slavcheva, Adriana (Hg.) (2014): Gesprochene Wissenschaftssprache. Korpusmethodische Fragen und empirische Analysen. (= Wissenschaftskommunikation 9). Heidelberg.

Garrett, Peter (2010): Attitudes to language. Cambridge. 
Gärtig, Anne-Kathrin/Plewnia, Albrecht/Rothe, Astrid (2010): Wie Menschen in Deutschland über Sprache denken. Ergebnisse einer bundesweiten Repräsentativerhebung zu aktuellen Spracheinstellungen. (= amades - Arbeitspapiere und Materialien zur deutschen Sprache 40). Mannheim.

Hagemann, Jörg/Klein, Wolf Peter/Staffeldt, Sven (Hg.) (2013): Pragmatischer Standard. (= Stauffenburg Linguistik 73). Tübingen.

Katerbow, Matthias/Werth, Alexander (Hg.) (2010): Moderne Regionalsprachen als multidimensionales Forschungsfeld. (= Germanistische Linguistik 210). Hildesheim.

Kehrein, Roland (2012): Regionalsprachliche Spektren im Raum. Zur linguistischen Struktur der Vertikale. (= Zeitschrift für Dialektologie und Linguistik - Beihefte 152). Stuttgart.

Kehrein, Roland/Lameli, Alfred/Rabanus, Stefan (Hg.) (2015): Regionale Variation des Deutschen. Projekte und Perspektiven. Berlin.

König, Katharina (2014): Spracheinstellungen und Identitätskonstruktion. Eine gesprächsanalytische Untersuchung sprachbiographischer Interviews mit Deutsch-Vietnamesen. (= Empirische Linguistik 2). Berlin.

König, Werner/Elspaß, Stephan/Möller, Robert (2015): dtv-Atlas deutsche Sprache. Orig.-Ausg., 18., durchges. u. korr. Aufl. München.

Lameli, Alfred/Purschke, Christoph/Kehrein, Roland (2008): Stimulus und Kognition. Zur Aktivierung mentaler Raumbilder. In: Linguistik Online 35, 3, S. 55-86.

Lenz, Alexandra N. (2003): Struktur und Dynamik des Substandards. Eine Studie zum Westmitteldeutschen (Wittlich/Eifel). (= Zeitschrift für Dialektologie und Linguistik - Beihefte 125). Stuttgart.

Liebscher, Grit/Dailey-O'Cain, Jennifer (2009): Language attitudes in interaction. In: Journal of Sociolinguistics 13, 2, S. 195-222.

Linell, Per (2005): The written language bias in linguistics. Its nature, origins and transformations. (= Routledge Advances in Communication and Linguistic Theory 5). London.

Löffler, Heinrich (2005): Wieviel Variation verträgt die deutsche Standardsprache? Begriffsklärung: Standard und Gegenbegriffe. In: Eichinger/Kallmeyer (Hg.), S. 7-27.

Moosmüller, Sylvia (2015): Methodisches zur Bestimmung der Standardaussprache in Österreich. In: Lenz, Alexandra N./Glauninger, Manfred Michael (Hg.): Standarddeutsch im 21. Jahrhundert. Theoretische und empirische Ansätze mit einem Fokus auf Österreich. (= Wiener Arbeiten zur Linguistik 1). Göttingen, S. 165-184.

Plewnia, Albrecht/Rothe, Astrid (2012): Sprache - Einstellungen - Regionalität. In: Eichinger, Ludwig M. et al. (Hg.): Sprache und Einstellungen. Spracheinstellungen aus sprachwissenschaftlicher und sozialpsychologischer Perspektive. (= Studien zur Deutschen Sprache 61). Tübingen, S. 9-118.

Purschke, Christoph (2010): Regionalsprachliches Wissen und Perzeption. Zur Konzeptionalisierung des Hessischen. In: Katerbow, Matthias/Werth, Alexander (Hg.): Moderne Regionalsprachen als multidimensionales Forschungsfeld. (= Germanistische Linguistik 210). Hildesheim u.a., S. 93-127.

Ransmayr, Jutta (2006): Der Status des österreichischen Deutsch an nichtdeutschsprachigen Universitäten. Eine empirische Untersuchung. (= Österreichisches Deutsch - Sprache der Gegenwart 8). Frankfurt a.M.

Schmidt, Jürgen Erich (2017): Vom traditionellen Dialekt zu den modernen deutschen Regionalsprachen. In: Deutsche Akademie für Sprache und Dichtung/Union der Deutschen Akademien der Wissenschaften (Hg.), S. 105-143. 
Sieber, Peter (2013): Probleme und Chancen der Diglossie - Einstellungen zu Mundarten und Hochdeutsch in der Deutschschweiz. In: Eriksson, Brigit/Luginbühl, Martin/Tuor, Nadine (Hg.): Sprechen und Zuhören - gefragte Kompetenzen? Überzeugungen zur Mündlichkeit in Schule und Beruf. (= Mündlichkeit 2). Bern, S. 106-136.

Spiekermann, Helmut (2005): Regionale Standardisierung, nationale Destandardisierung. In: Eichinger/Kallmeyer (Hg.), S. 100-125.

Steger, Hugo (1968): Über das Verhältnis von Sprachnorm und Sprachentwicklung in der deutschen Gegenwartssprache. In: Moser, Hugo (Hg.): Sprachnorm, Sprachpflege, Sprachkritik. Jahrbuch 1966/1967. (= Sprache der Gegenwart 2). Düsseldorf, S. 45-66.

Tophinke, Doris/Ziegler, Evelyn (2014): Spontane Dialektthematisierung in der Weblogkommunikation. In: Cuonz, Christina/Studler, Rebekka (Hg.): Sprechen über Sprache. Perspektiven und neue Methoden der Spracheinstellungsforschung. (= Stauffenburg Linguistik 81). Tübingen, S. 205-242.

Vergeiner, Philip (i.Vorb.): Konformität oder Freiheit? Zur sprachlichen Variation in globalisierten Räumen am Beispiel der Universität Salzburg (Arbeitstitel). Innsbruck. [Diss.].

Wandruszka, Mario (1979): Die Mehrsprachigkeit des Menschen. München.

Wiesinger, Peter (1997): Sprachliche Varietäten - gestern und heute. In: Stickel, Gerhard (Hg.): Varietäten des Deutschen. Regional- und Umgangssprachen. (= Jahrbuch des Instituts für Deutsche Sprache 1996). Berlin, S. 9-45. 